\title{
Thermoelastic equation of state and melting of Mg metal at high pressure and high temperature
}

Cite as: J. Appl. Phys. 127, 055903 (2020); https://doi.org/10.1063/1.5135649

Submitted: 12 November 2019 . Accepted: 19 January 2020 . Published Online: 05 February 2020

Alexandre Courac (D), Yann Le Godec, Vladimir L. Solozhenko (D), Nicolas Guignot, and Wilson A. Crichton
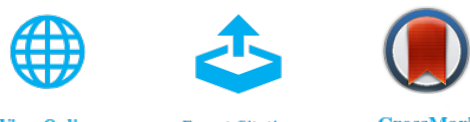

Export Citation

\section{ARTICLES YOU MAY BE INTERESTED IN}

Myths about new ultrahard phases: Why materials that are significantly superior to diamond in elastic moduli and hardness are impossible

Journal of Applied Physics 125, 130901 (2019); https://doi.org/10.1063/1.5082739

Acoustic and double elastic shock waves in single-crystal graphene

Journal of Applied Physics 127, 055101 (2020); https://doi.org/10.1063/1.5132925

Defect distribution in boron doped silicon nanostructures characterized by means of scanning spreading resistance microscopy

Journal of Applied Physics 127, 055703 (2020); https://doi.org/10.1063/1.5134558

Lock-in Amplifiers Find out more today

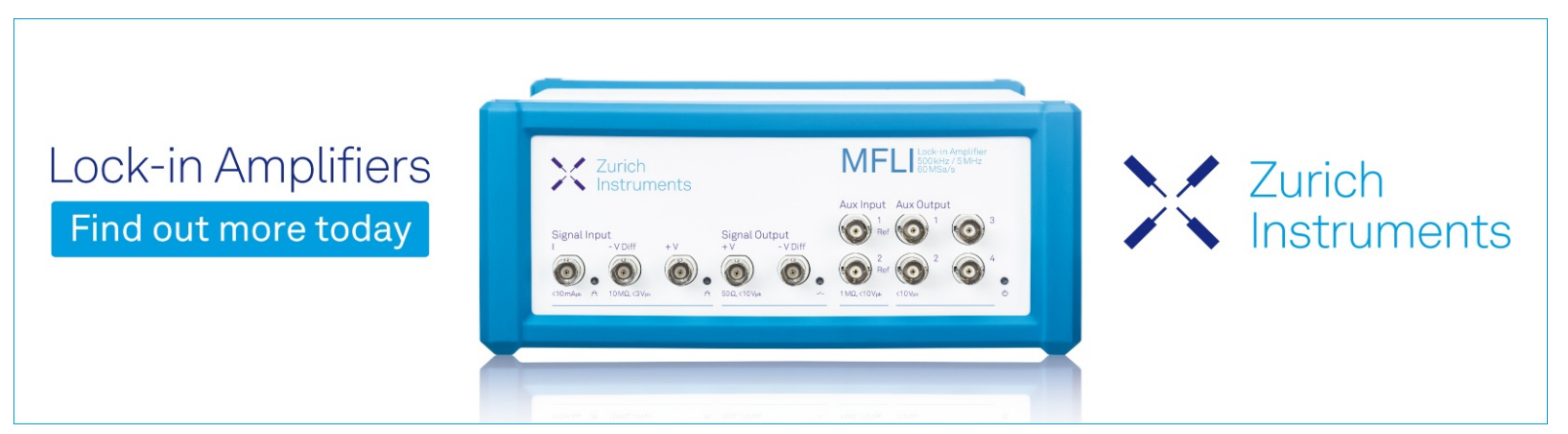

Zurich - Instruments 


\title{
Thermoelastic equation of state and melting of $\mathrm{Mg}$ metal at high pressure and high temperature
}

\author{
Cite as: J. Appl. Phys. 127, 055903 (2020); doi: 10.1063/1.5135649 \\ Submitted: 12 November 2019 . Accepted: 19 January 2020 . \\ Published Online: 5 February 2020
}

Alexandre Courac, ${ }^{1,2, a)}$ (D) Yann Le Godec, ${ }^{7}$ Vladimir L. Solozhenko, ${ }^{3}$ (D) Nicolas Guignot, ${ }^{4}$ and Wilson A. Crichton ${ }^{5}$

\author{
AFFILIATIONS \\ ${ }^{1}$ Institut de Minéralogie, de Physique des Matériaux et de Cosmochimie (IMPMC), Sorbonne Université, UMR CNRS 7590, \\ Muséum National d'Histoire Naturelle, IRD UMR 206, 75005 Paris, France \\ ${ }^{2}$ Institut Universitaire de France (IUF), 75005 Paris, France \\ ${ }^{3}$ LSPM-CNRS, Université Paris Nord, 93430 Villetaneuse, France \\ ${ }^{4}$ Synchrotron SOLEIL, 91192 Gif-sur-Yvette, France \\ ${ }^{5}$ ESRF-The European Synchrotron Facility, 71, avenue des Martyrs, 38000 Grenoble, France
}

a) Author to whom correspondence should be addressed: alexandre.courac@sorbonne-universite.fr

\begin{abstract}
The $p-V-T$ equation of state of magnesium metal has been measured up to $20 \mathrm{GPa}$ and $1500 \mathrm{~K}$ using both multianvil and opposite anvil techniques combined with synchrotron x-ray diffraction. To fit the experimental data, the model of Anderson-Grüneisen has been used with fixed parameter $\delta_{\mathrm{T}}$. The $300-\mathrm{K}$ bulk modulus of $B_{0}=32.5(1) \mathrm{GPa}$ and its first pressure derivative, $B_{0}{ }^{\prime}=3.73(2)$, have been obtained by fitting available data up to $20 \mathrm{GPa}$ to the Murnaghan equation of state. Thermal expansion at ambient pressure has been described using second order polynomial with coefficients $a=25(2) \times 10^{-6} \mathrm{~K}^{-1}$ and $b=9.4(4) \times 10^{-9} \mathrm{~K}^{-2}$. The parameter describing simultaneous pressure and temperature impact on the thermal expansion coefficient (and, therefore, volume) is $\delta_{\mathrm{T}}=1.5(5)$. The good agreement between fitted and experimental isobars has been achieved to relative volumes of 0.75 . The $\mathrm{Mg}$ melting observed by $\mathrm{x}$-ray diffraction and in situ electrical resistivity measurements confirms previous results and additionally confirms the $p-T$ estimations in the vicinity of melting.
\end{abstract}

Published under license by AIP Publishing. https://doi.org/10.1063/1.5135649

\section{INTRODUCTION}

Study of magnesium metal under high pressure is interesting not only from a fundamental point of view for searching new exotic matter state at extremely high pressures, ${ }^{1}$ but also for understanding of thermochemical properties of $\mathrm{Mg}$-bearing systems for synthesis of diamond ${ }^{2}$ and energetic materials ${ }^{3,4}$ at high pressure-high temperature (HPHT) conditions. The phase transformations in $\mathrm{Mg}$ have been studied at HPHT by Errandonea et $a .^{5}$ and Stinton et al. ${ }^{6}$ Above $1200 \mathrm{~K}$ in the $10-20 \mathrm{GPa}$ range, the formation of a double-hexagonal close-packed (dhcp) phase has been observed from an initial hcp structure. The dhcp phase may be recovered as metastable at room temperature and high pressure in a diamond anvil cell (at $\sim 8 \mathrm{GPa}$ ). At the same time, data $^{5,6}$ analysis shows that the volume change during hcp to dhcp transformation, if it exists, is close to zero.

Magnesium compressibility and thermal expansion have been previously measured by both in situ x-ray diffraction (XRD) and dilatometry (Table I). ${ }^{5-10}$ The most recent and accurate results give the bulk modulus $B_{0}=32.5(4) \mathrm{GPa}$ with its pressure derivative $B^{\prime}{ }_{0}=4.05(5) .{ }^{6}$ The linear thermal expansion $[L(T)$ at $0.1 \mathrm{MPa}]$ of $\mathrm{Mg}$ up to the melting temperature well follows the square polynomial with thermal expansion coefficient $\alpha_{\mathrm{L}}=25 \times 10^{-6} \mathrm{~K}^{-1}$ and its first temperature derivative $\alpha_{\mathrm{L}}^{\prime}=18.8 \times 10^{-9} \mathrm{~K}^{-2}$ (at $273 \mathrm{~K}$ ). ${ }^{8}$ The slightly nonisotropic lattice expansion has been reported in Ref. 9

Previously, the $p-V-T$ equation of state (EoS) was only reported in Ref. 5 up to $19 \mathrm{GPa}$ and melting temperatures; however, the proposed analytical expression for the $p-V$ - $T$ EoS suffers from thermodynamic inconsistency due to the linear extrapolation of temperature dependence of the bulk modulus (e.g., either crossing isotherms become possible or negative bulk modulus). At the same time, the relatively small number of data was not sufficient for fitting of the parameters describing thermal dependencies of bulk modulus and thermal expansion. The thermodynamically consistent model with a smaller number of assumptions and fitting parameters is required 
TABLE I. Pressure $p$, temperature $T$, and relative volume $V I V_{0}$ data $\left(V_{0}=13.98 \mathrm{~cm}^{3} \mathrm{~g}^{-1}\right)$ from previous reports. ${ }^{5,6}$

\begin{tabular}{|c|c|c|c|c|c|}
\hline$p, \mathrm{GPa}$ & $T, \mathrm{~K}$ & $V / V_{0}$ & $p, \mathrm{GPa}$ & $T, \mathrm{~K}$ & $V / V_{0}$ \\
\hline \multicolumn{6}{|c|}{ Data from Ref. 5} \\
\hline 0 & 300 & 0.9957 & 12.07 & 1077 & 0.8483 \\
\hline 0 & 300 & 0.9978 & 12.07 & 1127 & 0.8519 \\
\hline 0 & 300 & 1.0050 & 12.07 & 1127 & 0.8562 \\
\hline 0 & 300 & 0.9835 & 12.08 & 977 & 0.8154 \\
\hline 0.65 & 300 & 0.9692 & 12.4 & 927 & 0.8097 \\
\hline 3.77 & 300 & 0.8719 & 12.4 & 927 & 0.8097 \\
\hline 4.53 & 300 & 0.8927 & 12.5 & 1377 & 0.8218 \\
\hline 4.98 & 300 & 0.9105 & 12.82 & 877 & 0.8104 \\
\hline 5.25 & 300 & 0.8898 & 13.1 & 1247 & 0.8233 \\
\hline 7.02 & 877 & 0.9341 & 13.65 & 677 & 0.8068 \\
\hline 8.05 & 300 & 0.8447 & 13.7 & 1177 & 0.7954 \\
\hline 8.6 & 1127 & 0.9206 & 14.08 & 1047 & 0.7546 \\
\hline 9.64 & 1277 & 0.8826 & 14.38 & 477 & 0.7939 \\
\hline 10.5 & 1477 & 0.8927 & 14.6 & 1047 & 0.7675 \\
\hline 10.7 & 1427 & 0.8898 & 14.75 & 300 & 0.7811 \\
\hline 11.1 & 1377 & 0.8798 & 15.3 & 877 & 0.7875 \\
\hline 11.3 & 1327 & 0.8733 & 15.8 & 677 & 0.7789 \\
\hline 11.3 & 1327 & 0.8691 & 16.3 & 477 & 0.7646 \\
\hline 11.56 & 1277 & 0.8619 & 16.8 & 300 & 0.7560 \\
\hline 11.8 & 1227 & 0.8633 & 17.65 & 300 & 0.7525 \\
\hline 11.8 & 1227 & 0.8612 & 18.5 & 577 & 0.7625 \\
\hline 12.06 & 1177 & 0.8483 & 18.6 & 300 & 0.7596 \\
\hline 12.07 & 1027 & 0.8483 & 18.5 & 577 & 0.7625 \\
\hline 12.07 & 1077 & 0.8505 & & & \\
\hline \multicolumn{6}{|c|}{ Data from Ref. $6^{\mathrm{a}}$} \\
\hline $3.6(6.5)$ & 300 & 0.850 & $5.0(6.5)$ & 1170 & 0.898 \\
\hline $4.4(6.5)$ & 1018 & 0.866 & $5.6(6.5)$ & 1315 & 0.907 \\
\hline
\end{tabular}

${ }^{\text {a The }} V / V_{0}$ values were evaluated from Fig. 4 of Ref. 6, pressure at $300 \mathrm{~K}$ (value in parences) was reestimated using $\mathrm{Mg}$ equation of state ${ }^{6}$ and better agree with observed relative volumes of $\mathrm{Mg}$ and with the fact that at $1315 \mathrm{~K}$ Mg still remain solid. ${ }^{6}$

to treat such data, for example, the model ${ }^{11-13}$ with constant Anderson-Grüneisen coefficient $\delta_{\mathrm{T}}{ }^{14,15}$

In the present work, the $p-V-T$ equation of state of $\mathrm{Mg}$ has been studied by in situ synchrotron $\mathrm{x}$-ray diffraction (XRD) up to $20 \mathrm{GPa}$ and melting temperatures. The analytical expression for the $p-V-T$ equation of state combines previously reported $p-V$ and $V-T$ data (at $300 \mathrm{~K}$ and $0.1 \mathrm{MPa}$, respectively) and one fitting parameter, the Anderson-Grüneisen coefficient $\delta_{\mathrm{T}}{ }^{14,15}$

\section{EXPERIMENTAL}

Chemically pure Mg powder (Alfa Aesar, 99.99 at. \%) has been used as a starting material. The experiments have been conducted in $\mathrm{MgO}$ capsules with various pressure media. The reaction with magnesium occurs at quite high temperatures and is observable by XRD after melting. In the solid state, the intergrain interaction is quite slow and can be observed only during many hour heating. ${ }^{4}$ Only in a limited numbers of experiments, we could observe the thermal expansion up to melting.
The Paris-Edinburgh (PE) press at beamline PSYCHÉ of synchrotron SOLEIL was used for obtaining in situ data at high temperatures in the 5.0-6.5 GPa range. Opposite anvils with standard boron-epoxy gasket (pressure medium) were employed to pressurize the sample. A tubular graphite resistive furnace allowed heating up to $1500 \mathrm{~K}$ under high pressure. The $\mathrm{Mg}-\mathrm{C}$ mixtures were loaded into an $\mathrm{MgO}$ capsule in order to isolate the sample from the heater. Pressure and temperature estimations were made using $300-\mathrm{K}$ equations of states of $\mathrm{Mg}^{6}$ and $\mathrm{MgO}^{16}$ and temperature calibration curve obtained using Si melting point at HPHT. ${ }^{17,18}$ The phase transformations were observed by energy-dispersive $\mathrm{x}$-ray diffraction $\left[2 \theta=8.0(2)^{\circ}\right]$. The system was calibrated using an Au standard.

The high-pressure experiments at 1.5-8 GPa (according to the $\mathrm{hBN}$ equation of state $)^{19}$ with direct temperature measurements were carried out using the multianvil $\mathrm{x}$-ray system MAX80 ${ }^{20}$ with anvils of tungsten carbide. The diffraction measurements were performed in the energy-dispersive mode at beamline F2.1 (HASYLAB-DESY, Hamburg). The $\mathrm{Mg}+\mathrm{B}$ (amorphous) and $\mathrm{Mg}+\mathrm{hBN}$ samples were prepared in a glovebox with dry argon. X-ray patterns were collected on a Canberra solid state Ge-detector with a fixed Bragg angle $2 \theta=9.96(2)^{\circ}$. The details of experiments and high-pressure setup have been described elsewhere. ${ }^{21}$ The temperature of the highpressure cell was controlled by a Eurotherm PID regulator within $2 \mathrm{~K}$. The sample temperature was measured by a Pt $10 \% \mathrm{Rh}-\mathrm{Pt}$ thermocouple with its junction $300 \mu \mathrm{m}$ below the sample region under study (correction for the pressure effect on the thermocouple emf was made using the data of Li et $a l .^{22}$ ). The primary polychromatic synchrotron beam collimated down to $60 \times 100 \mu \mathrm{m}^{2}$ was perpendicular to the vertical axis of the sample chamber. The energy-dispersive diffraction patterns were collected in situ at a constant pressure in an "autosequence" mode in the course of a linear heating (or cooling) at a rate of $30 \mathrm{~K} \mathrm{~min}^{-1}$; the time of data collection for each pattern was $30 \mathrm{~s}$.

In situ experiments at $8-20 \mathrm{GPa}(\mathrm{Mg}+$ glassy carbon) were performed using the 20MN Voggenreiter press at beamline ID06 of the European Synchrotron Radiation Facility (ESRF). ${ }^{23}$ The $\mathrm{Mg}+\mathrm{C}$ mixture was ground in a ceramic mortar inside a highpurity Ar glovebox and loaded into an $\mathrm{MgO}$ capsule. The sample was then introduced (under Ar atmosphere) into a 10/5 multianvil assembly (MgO: $\mathrm{Cr}_{2} \mathrm{O}_{3}$ octahedron with $10 \mathrm{~mm}$ side compressed by eight WC cubic anvils with $5 \mathrm{~mm}$-side triangular truncations), equipped with graphite or Re furnaces. Temperatures and pressures were monitored using the $300-\mathrm{K}$ equations of state of $\mathrm{MgO}^{16}$ and $\mathrm{Mg}^{6}$ in parallel with estimation from previous calibration curves ( $\mathrm{Si}$ EoS and melting $)^{17,18}$ and W-Re thermocouples. Monochromatic $x$-ray diffraction data were taken using the beam with wavelength of $0.3757 \AA(33 \mathrm{keV})$. The beam was collimated to define a horizontal beam size of $\sim 1 \mathrm{~mm}$ to ensure that the whole sample was probed. Diffraction patterns were collected on an azimuthally scanning Detection Technology X-Scan c series GOS linear detector (the data collected continuously, at fixed azimuth).

The absence of remarkable broadening has been used as criterion of reaching the quasihydrostatic conditions and was typically observed above $600 \mathrm{~K}$. Only these parts of experimental quasi-isobars have been used for fit to the equation of state. (In practice, pressure changes during heating, and the sample is not at the pressure estimated before heating.) The $p, T$-values were refined using 
crosscalibration $(\mathrm{MgO}, \mathrm{hBN})$ or a pressure gauge compound with thermocouple. The data on thermal expansion of magnesium at given pressure were in some cases limited to the onset of reaction with a pressure medium (typically at $\sim 1500 \mathrm{~K}$ for $\mathrm{Mg}+\mathrm{C}$ mixture and $\sim 1400 \mathrm{~K}$ for $\mathrm{Mg}+\mathrm{B}$ mixture $^{21}$ in the pressure range under study). The reaction(s) at high temperatures and/or melting precluded collection of data on cooling and upon decompression.

The error estimation when studing an EoS is always a delicate task. In general, the relative error of volumes is the smallest, $\delta V / V<0.1 \%$, due to the high precicion of XRD data. The lowest pressure error of our data can be estimated as $\delta p \sim 0.2 \mathrm{GPa}$ (only these data can be found in Table II) at high temperatures. However, it can rise up to $\delta p \sim 2 \mathrm{GPa}$ (our observations in multianvil) and even higher in some cases (see following analysis of the diamond anvil cell and multianvil data from Refs. 5 and 6, Table I) at the initial stages of heating due to the lack of mechanical equilibrium between calibrants or if the cold (initial) pressure is used (such points were not used for fitting). As for the temperature, the highest precision can be obtained by thermocouple, $\delta T<1 \mathrm{~K}$, taking into acount the pressure dependence of thermocouple electricam motion force ${ }^{22}$ and distance between thermocouple joint and sample area probed by XRD. In our case, only the data obtained at HASYLAB meets such criterion with $\delta T \sim 1 \mathrm{~K}$. The highest estimate of $\delta T$ can be given by typical reproductibility of

TABLE II. Pressure $p$, temperature $T$, and relative volume $V / V_{0}$ data $\left(V_{0}=13.98 \mathrm{~cm}^{3} \mathrm{~g}^{-1}\right)$ from our experiments. (Estimations of typical measurement errors are given in the Experimental section.)

\begin{tabular}{lccccc}
\hline \hline$p, \mathrm{GPa}$ & $T, \mathrm{~K}$ & $V / V_{0}$ & $p, \mathrm{GPa}$ & $T, \mathrm{~K}$ & $V / V_{0}$ \\
\hline \multicolumn{4}{l}{ HASYLAB-DESY data } & (errors: $\delta V / V$ & $\sim 0.1 \%, \delta p \sim 0.2 \mathrm{GPa}, \delta T \sim 1 \mathrm{~K})$ \\
1.5 & 587 & 0.9971 & 7.0 & 775 & 0.8866 \\
2.3 & 877 & 0.9978 & 7.0 & 675 & 0.8781 \\
2.7 & 1047 & 0.9950 & 6.0 & 300 & 0.8697 \\
2.7 & 977 & 0.9964 & 6.0 & 875 & 0.9123 \\
7.0 & 300 & 0.8561 & 6.0 & 1025 & 0.9238
\end{tabular}

SOLEIL data (errors: $\delta V / V \sim 0.1 \%, \delta p \sim 0.2 \mathrm{GPa}, \delta T \sim 10 \mathrm{~K}$ )

$\begin{array}{rrrrrr}4.9 & 300 & 0.8871 & 5.0 & 300 & 0.8851 \\ 5.2 & 400 & 0.8848 & 5.3 & 600 & 0.8977 \\ 5.5 & 503 & 0.8893 & 5.2 & 750 & 0.9127 \\ 5.9 & 601 & 0.8904 & 5.7 & 930 & 0.9173 \\ 6.0 & 700 & 0.8932 & 5.6 & 950 & 0.9231 \\ 6.0 & 804 & 0.8988 & 5.5 & 1150 & 0.9443 \\ 6.0 & 902 & 0.9110 & 5.9 & 1250 & 0.9396\end{array}$

$\begin{array}{lll}6.0 & 937 & 0.9133\end{array}$

$\begin{array}{lll}6.0 & 972 & 0.9167\end{array}$

$\begin{array}{lll}6.1 & 1007 & 0.9194\end{array}$

$6.0 \quad 1056 \quad 0.9225$

ESRF data (errors: $\delta V / V \sim 0.1 \%, \delta p \sim 0.2 \mathrm{GPa}, \delta T \sim 10 \mathrm{~K}$ )

$\begin{array}{llllll}8.0 & 925 & 0.8791 & 17.0 & 300 & 0.7535\end{array}$

$\begin{array}{llllll}8.4 & 1070 & 0.8881 & 18.0 & 800 & 0.7623\end{array}$

$\begin{array}{llllll}8.8 & 1355 & 0.9075 & 18.1 & 1300 & 0.7830\end{array}$

$\begin{array}{llllll}9.0 & 1450 & 0.9121 & 18.5 & 1025 & 0.7708\end{array}$

$\begin{array}{lll}17.0 & 927 & 0.7824\end{array}$

\begin{tabular}{lll}
17.5 & 1127 & 0.7817 \\
\hline
\end{tabular}

calibration curve $(\delta T \sim 50-100 \mathrm{~K})$, while using $p-V$ - $T$ EoS of calibrants allow decreasing the error to $\delta T \sim 10-20 \mathrm{~K}$ (the case of Table II for ESRF and SOLEIL data).

In order to avoid the possible systematic errors in the estimation of melting points by XRD (recrystallization and single crystal growth may also cause the apparent disappearance of diffraction rings) at various pressures, we also monitored the onset of melting via electrical measurements of the furnace assembly (Fig. 1). In fact, when using the solid $\mathrm{Mg}+$ glassy $\mathrm{C}$ mixture in contact with graphite heater, the resistance of heater remains very close to that of heater alone. On increasing temperature, a trace dominated by a typical semiconductor furnace is evident [Fig. 1(b)]. At melting, $\mathrm{Mg}$ penetrates the whole assembly and causes a significant drop in resistance enabling us to pinpoint the onset clearly. Similar method has been recently used for the melting curve measurement of $\mathrm{Na}_{4} \mathrm{Si}_{4}{ }^{24}$ The knowledge of the Mg melting curve also allowed us to refine the pressure or temperature values in some cases.

\section{COMPUTATIONAL METHOD}

For the equation of state data fitting, we have used integrated form of the Anderson-Grüneisen equation. ${ }^{14,15}$ In previous works, ${ }^{11-13}$ we have shown that this equation, which takes into account the pressure dependence of thermal expansion coefficient $\alpha$ only through the volume change, i.e.,

$$
\alpha(p, T)=\alpha(0, T)\left[\frac{V(p, T)}{V(0, T)}\right]^{\delta_{T}},
$$

can be integrated (under the assumption that $\delta_{\mathrm{T}}$ is constant over the studied $p$ - $T$ domain) to

$$
V(p, T)=\left[V(0, T)^{-\delta_{T}}+V(p, 300)^{-\delta_{T}}-V(0,300)^{-\delta_{T}}\right]^{-1 / \delta_{T}},
$$

where thermal expansion [i.e., $V(0, T)$ at $0.1 \mathrm{MPa}$ ] and isothermal compression [i.e., $V(p, 300)$ at $300 \mathrm{~K}$ ] can be presented in any analytical form, e.g., polynomial

$$
\begin{aligned}
V(0, T)=V(0,300)[1+ & a(T-273)+b(T-273)^{2} \\
& \left.-a(300-273)+b(300-273)^{2}\right]^{3},
\end{aligned}
$$

with $a=\alpha_{\mathrm{L}}$ and $b=0.5 \times \alpha_{\mathrm{L}}^{\prime}$ and the Murnaghan (or any other) equation of state

$$
V(p, 300)=V(0,300)\left(1+B_{0}^{\prime} p / B_{0}\right)^{-1 / B_{0}} .
$$

Thus, a set of parameters needed to describe an EoS using Eqs. (2)-(4) is $V_{0} \equiv \mathrm{V}(0,300) \equiv M / \rho_{0}, B_{0}, B_{0}{ }^{\prime}, a, b$, and $\delta_{\mathrm{T}}{ }^{12}$ Such form of the EoS, Eq. (2), allows one to easily approximate the $V(p, T)$ in the domain of interest for various objectives such as $p-T$ refinement or phase equilibria calculation. , $11-13,25^{-2}$

\section{RESULTS AND DISCUSSION}

At ambient conditions, $\mathrm{Mg}$ has a hexagonal structure [space group $P 6_{3} / m m c$ (No. 194)] with unit cell parameters $a=3.209(2) \AA$ 


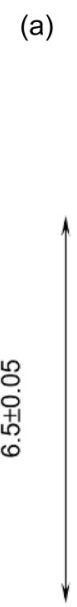

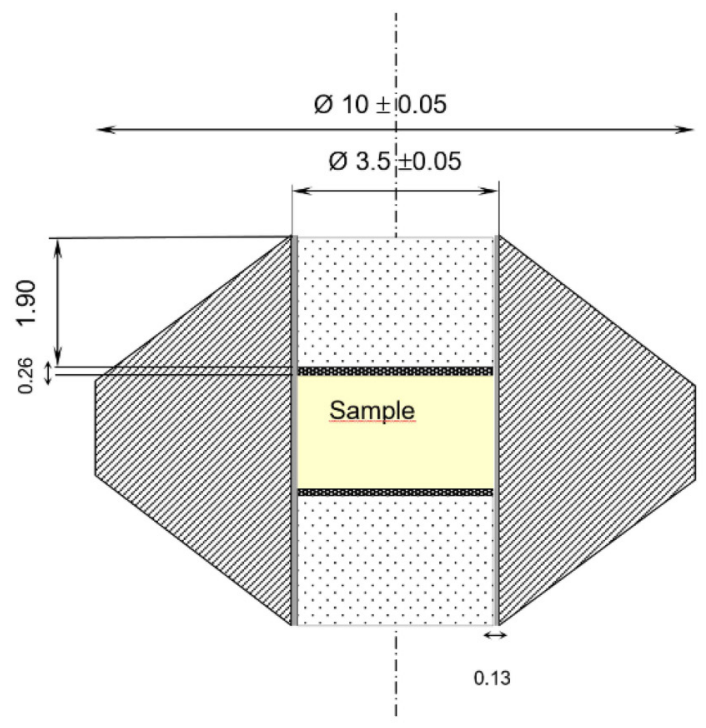

Pyrophyllite

Grafoil

Graphite or grafoil

$\mathrm{Al}_{2} \mathrm{O}_{3}$

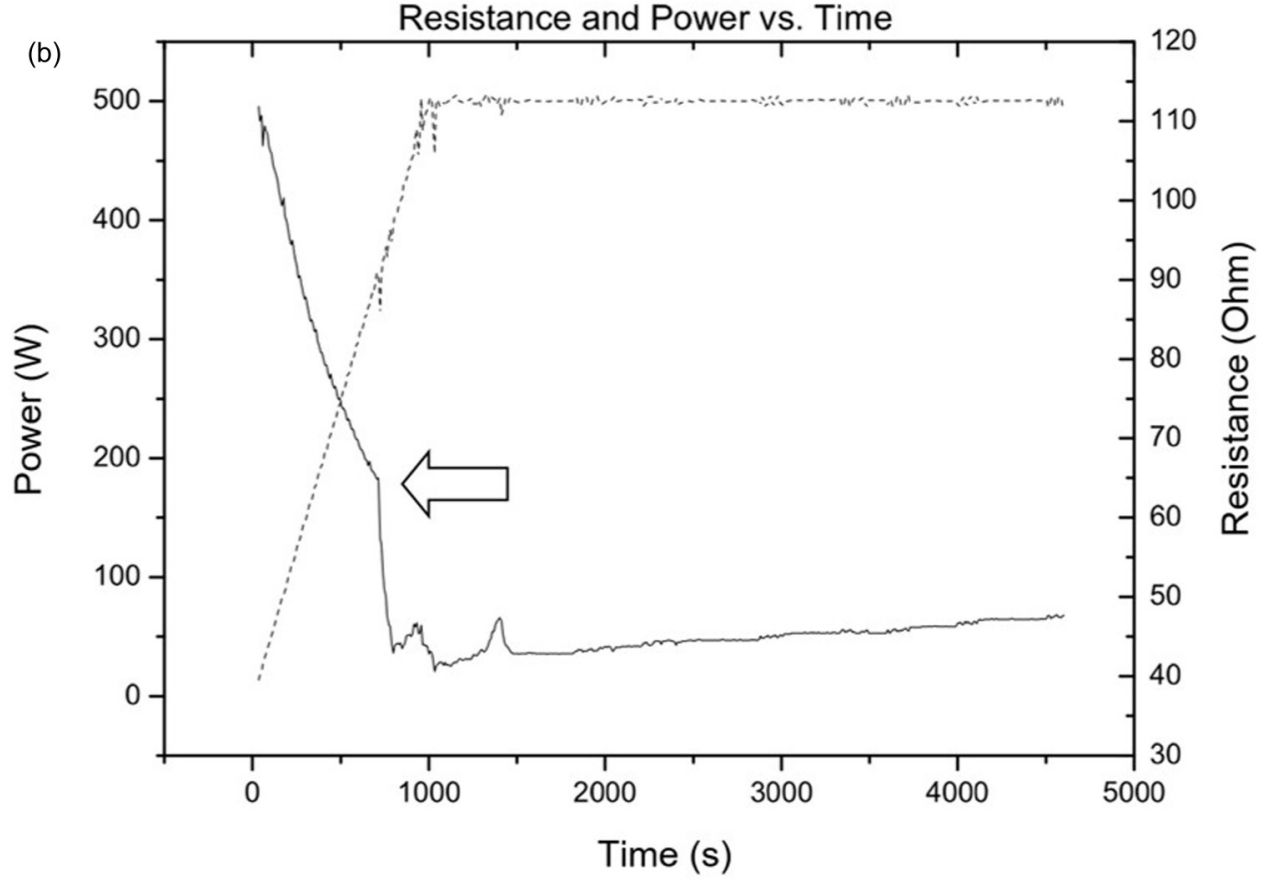

FIG. 1. (a) High-pressure PE cell $10 / 3.5$ for the electrical measurements of the (sample + heater) system (lengths are in $\mathrm{mm}$ ). (b) In situ furnace characteristics during power ramping (dotted line, left scale) of furnace containing $\mathrm{Mg}+\mathrm{C}$ mixture at $5 \mathrm{GPa}$. The resistance drop (solid line, right scale) at the arrow, corresponding to $1200 \mathrm{~K}$, is coincident with Mg melting.

and $c=5.211(3) \AA$. The synchrotron $\mathrm{x}$-ray diffraction patterns of $\mathrm{Mg}$ show three lines, i.e., 100,002 , and 101 , that were used to establish the molar volume $V(p, T)$ at HPHT conditions. The in situ $\mathrm{XRD}$ data on thermal expansion at given pressure (quasi-isobars) and observation of melting (disappearance of solid $\mathrm{Mg}$ reflections, appearance of characteristic halo of liquid) are shown in Fig. 2.

The $p-V$ - $T$ data (Tables I and II) of our experiments and from Refs. 5 and 6 are plotted in Fig. 3(a) (symbols) as compared with calculated isobars. Each color (symbols correspond to experimental points) covers the possible relative volume values $V / V_{0}$ at different temperatures for a 2-GPa pressure range (ten in total, covering total pressure domain of available data from $0.1 \mathrm{MPa}$ to $20 \mathrm{GPa}$ ). The pressure change during heating was not significant above $600 \mathrm{~K}$ but can be up to $2 \mathrm{GPa}$ higher or lower than before heating ("cold compression").

The best fit of experimental data has been obtained using the $300-\mathrm{K}$ equation of state of Stinton et al. ${ }^{6}$ [coefficients for Eq. (4) are $B_{0}=32.5(2) \mathrm{GPa}, B^{\prime}{ }_{0}=3.73(2)$, reproducing the data ${ }^{6}$ up to 

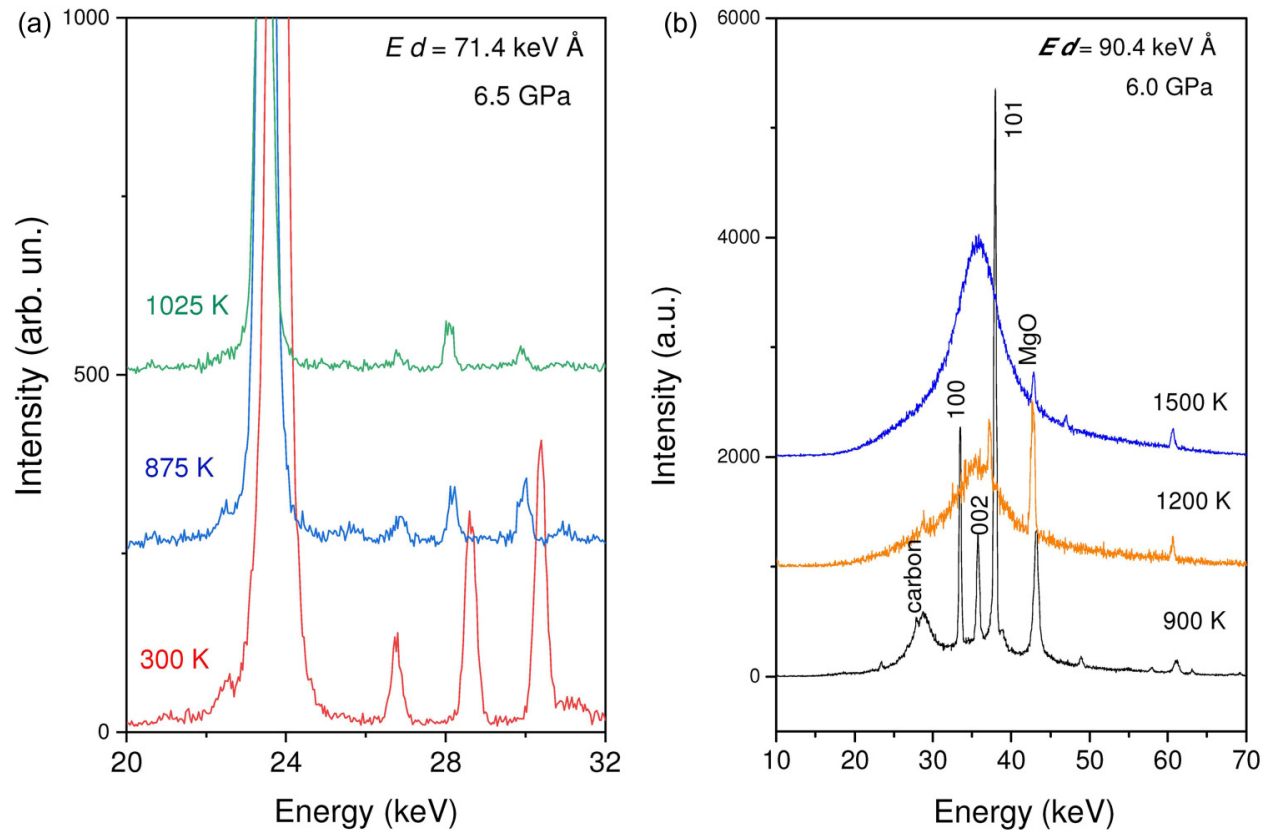

FIG. 2. In situ energy-dispersive XRD data on equation of state and melting of $\mathrm{Mg}$ taken at HASYLAB-DESY (a) and at PSYCHÉ beamline at SOLEIL (b).

$25 \mathrm{GPa}$ to high accuracy of relative volume, i.e., with uncertainty $\delta$ $\left.\left(V / V_{0}\right)<10^{-3}\right]$. The thermal expansion has been taken into account by the analytical expression, valid up to melting temperature [Eq. (3) with parameters $a=25 \times 10^{-6} \mathrm{~K}^{-1}$ and $b=9.4 \times 10^{-9} \mathrm{~K}^{-2}$ ]. As a guide for eyes to judge the quality of fit, we use the color match between theoretical domains and symbols in Fig. 3(a). The parameter $\delta_{\mathrm{T}}=1.5(5)$ gives the best fit for the $p-V-T$ experimental data.
Isothermal Anderson-Grüneisen parameter $\delta_{\mathrm{T}}$ for $\mathrm{Mg}$ has been previously evaluated by two alternative methods, ${ }^{26} \delta_{\mathrm{T}}=1.66$ using experimental data on the $T$-dependence of elastic constants ${ }^{27}$ and $\delta_{\mathrm{T}}=2.69$ from theoretical lattice dynamic study. ${ }^{28}$ One can see reasonable agreement of our $\delta_{\mathrm{T}}$ value obtained by the EoS fit with elastic measurements. ${ }^{26,27}$ The values of $\delta_{\mathrm{T}}=1.0$ and 2.0 also give reasonable agreement of all available experimental data (Tables I and II).

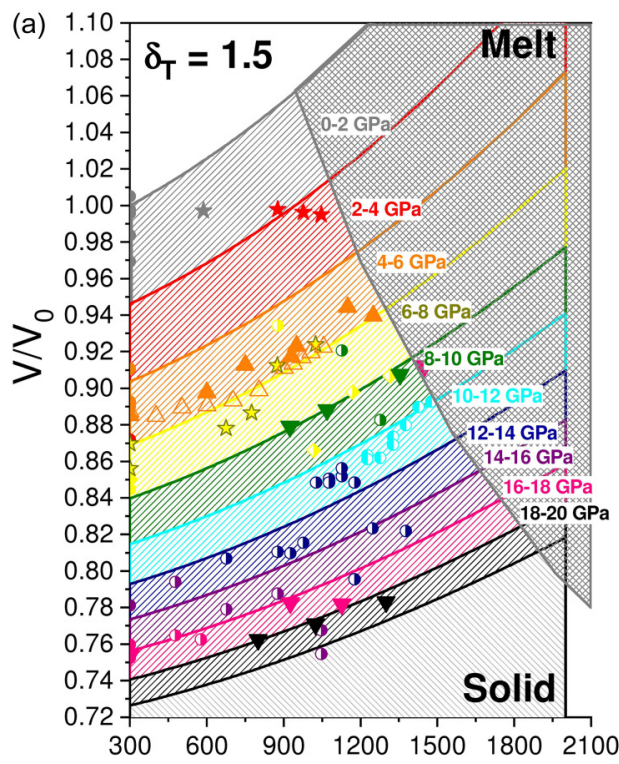

Temperature, $\mathrm{K}$

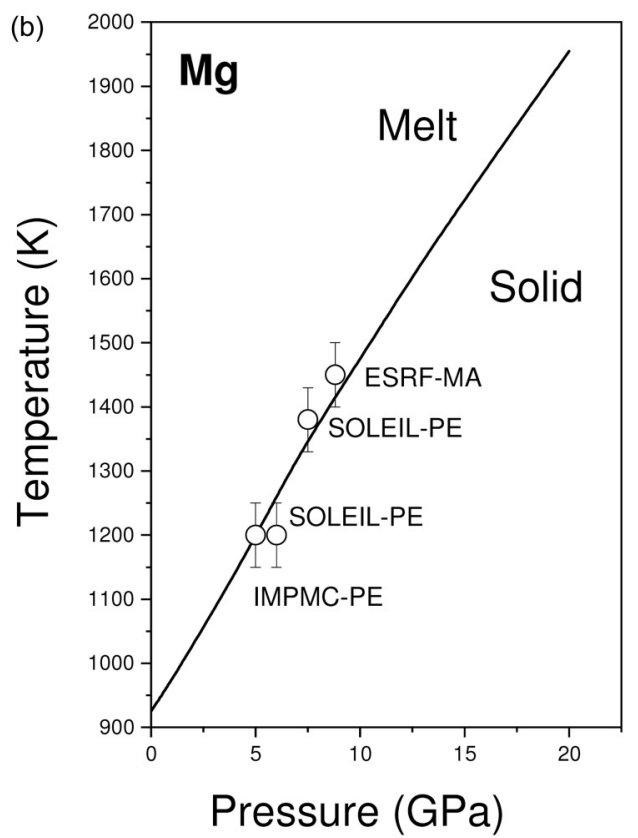

FIG. 3. (a) Theoretical isobars (2-GPa step, colored) as compared to experimental data. Grey ${ }^{5}$ grid area show the domain of melted $\mathrm{Mg}$ (with some points of the solid Mg while coexisting with liquid). Small colored circles represent the data from Refs. 5 and 6 (O) and $\mathbf{O})$, the corresponding 2-GPa domains (limited by theoretical isobar curves according to Eq. 2 and melting curve) are given with the same color. Our data is presented with following symbols, depending on the synchrotron source: $\mathbf{\nabla}$-ESRF, $\star$-HASYLAB-DESY and $\mathbf{\nabla}, \Delta$-SOLEIL. (b) Melting curve of $\mathrm{Mg}$ up to $20 \mathrm{GPa}$. Continuous curve corresponds to previous reports, while $O$ symbols show melting observed by electrical resistance (IMPMC) and XRD-(SOLEIL, ESRF). 


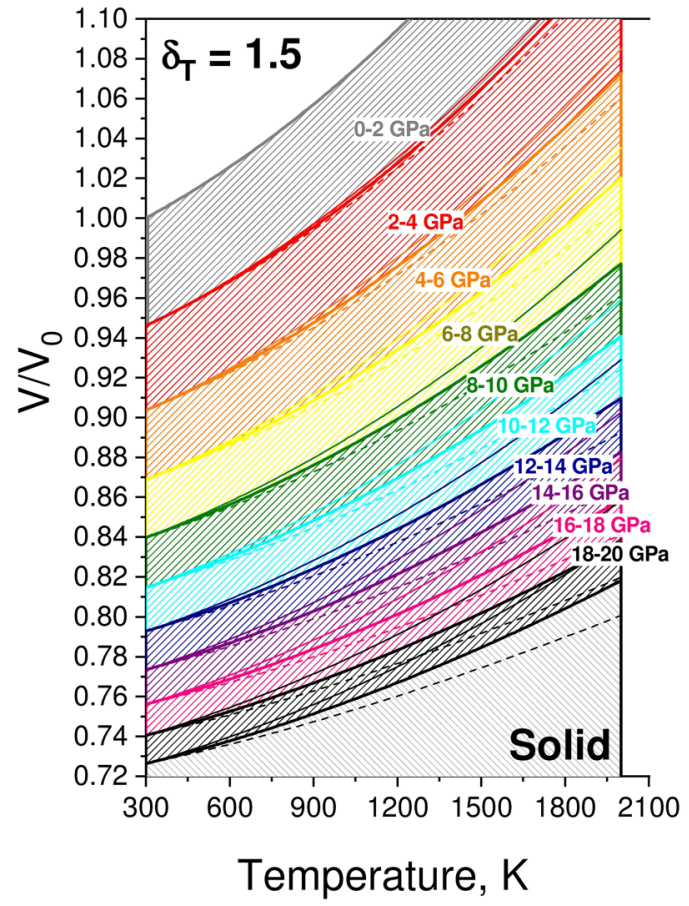

FIG. 4. Theoretical isobars (2-GPa step, colored) corresponding to $\delta_{\mathrm{T}}=1.5$ (thick solid lines), $\delta_{\top}=1.0$ (thin solid lines), and $\delta_{\top}=2$ (thin dashed lines).

Figure 4 shows the set of isobars (from $0.1 \mathrm{MPa}$ up to $20 \mathrm{GPa}$ ) for $\delta_{\mathrm{T}}=1.0,1.5$, and 2.0. Significant discrepancy is observed only for very high temperatures, where solid $\mathrm{Mg}$ does not exist.

It is also interesting to compare the $\mathrm{EoS}$ of $\mathrm{Mg}$ with $\mathrm{Ca}$, another alkaline metal stable in fcc (up to $700-750 \mathrm{~K}$ ), and bbc (up to melting) structures in a similar $p-T$ range. ${ }^{29}$ The bulk moduli of both $\mathrm{Ca}$ allotropes are smaller by a factor of two, while thermal expansion is just slightly smaller than corresponding values for fcc $\mathrm{Ca}$.

The in situ observations of melting at different synchrotron facilities (Fig. 2) and by resistance measurements [Fig. 1(b)] are presented at Fig. 3(b). Our experimental value of the zero-pressure melting slope is $\mathrm{d} T / \mathrm{d} p=60(5) \mathrm{K} \mathrm{GPa}^{-1}$, in agreement with previous resistivity measurements, of $60(2) \mathrm{K} \mathrm{GPa}^{-1}$. ${ }^{30}$ At higher pressure, the melting slope decrease down to $38(4) \mathrm{K} \mathrm{GPa}^{-1}$ at $\sim 10 \mathrm{GPa}$ according to our estimations from the data reported in Ref. 30. Such a noticeable decrease is often due to the higher compressibility of liquid phase as compared to solid.

The melting heat of $\mathrm{Mg}$ is $\Delta H_{0}=8.5 \mathrm{~kJ} \mathrm{~mol}^{-1} .^{31}$ Liquid density at melting temperature and ambient pressure is $\rho_{\mathrm{m}}=1.590(1) \mathrm{g} \mathrm{cm}^{-3}$ with linear negative thermal coefficient $-\mathrm{d} \rho_{\mathrm{m}} / \mathrm{d} T=2.647 \times 10^{-4} \mathrm{~g} \mathrm{~cm}^{-3} \mathrm{~K}^{-1}$ up to boiling temperature at $1390 \mathrm{~K}^{32}$ The room-temperature density at ambient pressure of solid $\mathrm{Mg}$ is $\rho_{0}=1.737 \mathrm{~g} \mathrm{~cm}^{-3}$, which gives the density estimation of $1.639 \mathrm{~g} \mathrm{~cm}^{-3}$ at melting point $(923 \mathrm{~K})$ according to our EoS. The value of melting volume is thus $\Delta V_{0}=+0.456(9) \mathrm{cm}^{3} \mathrm{~mol}^{-1}$, and the estimate for the melting curve slope is $\mathrm{d} T / \mathrm{d} p=47(2) \mathrm{K} \mathrm{GPa}^{-1}$, which is in satisfactory agreement (22\% accuracy) with observed value of $60 \mathrm{~K} \mathrm{GPa}^{-1} \cdot{ }^{23}$ Alternative estimates of $\mathrm{Mg}$ liquid density ${ }^{33}$ $\left(\rho_{\mathrm{m}}=1.584 \mathrm{~g} \mathrm{~cm}^{-3},-\mathrm{d} \rho_{\mathrm{m}} / \mathrm{d} T=2.34 \times 10^{-4} \mathrm{~g} \mathrm{~cm}^{-3} \mathrm{~K}^{-1}\right)$ give better agreement: volume is $\Delta V_{0}=+0.51(2) \mathrm{cm}^{3} \mathrm{~mol}^{-1}$, and the estimate for the melting curve slope is $\mathrm{d} T / \mathrm{d} p=53(2) \mathrm{K} \mathrm{GPa}^{-1}$, i.e., only $11 \%$ below the experimental estimate.

\section{CONCLUSIONS}

Finally, the $p-V-T$ equation of state of metallic $\mathrm{Mg}$ has been studied up to $20 \mathrm{GPa}$ and $1500 \mathrm{~K}$ using different high-pressure apparatuses and synchrotron radiation facilities. The model describing analytical expression for the temperature and pressure dependencies of volume is consistent with EoS parameters previously derived from both $300 \mathrm{~K}$ and $0.1 \mathrm{MPa}$ measurements, and the constant value of Anderson-Grüneisen parameter $\delta_{\mathrm{T}}=1.5(5)$ is appropriate to describe $V(p, T)$ all over the $p$ - $T$ range under study (to compression of 0.75 ). The melting $p$ - $T$ data by both XRD and electrical measurements are consistent with previous reports.

\section{ACKNOWLEDGMENTS}

A.C. and Y.L.G. acknowledge Agence Nationale de Recherche for financial support (Project No. ANR-17-CE08-0038). We acknowledge synchrotrons ESRF, DESY, and SOLEIL for provision of synchrotron radiation facilities. The in situ XRD experiments were performed (1) on beamline ID06-LVP at the European Synchrotron Radiation Facility (ESRF), Grenoble, France (allocated beamtime CH-5431); (2) on F2.1 beamline at HASYLAB-DESY, Hamburg, Germany; and (3) on beamline PSYCHÉ at SOLEIL, Gif-sur-Yvette, France. We are grateful to Dr. K. Spector at the ESRF for providing assistance in using beamline ID06-LVP. The authors also acknowledge Dr. H. Moutaabbid and Mr. I. Touloupas for assistance in highpressure experiments at IMPMC.

\section{REFERENCES}

${ }^{1}$ P. Li, G. Gao, Y. Wang, and Y. Ma, J. Phys. Chem. C 114(49), 21745-21749 (2010). .

${ }^{2}$ O. O. Kurakevych, Y. Le Godec, T. A. Strobel, D. Y. Kim, W. A. Crichton, and J. Guignard, J. Phys. Chem. C 118(15), 8128-8133 (2014).

${ }^{3}$ O. O. Kurakevych, T. A. Strobel, D. Y. Kim, and G. D. Cody, Angew. Chem. Int. Ed. 52(34), 8930-8933 (2013).

${ }^{4}$ T. A. Strobel, O. O. Kurakevych, D. Y. Kim, Y. Le Godec, W. Crichton, J. Guignard, N. Guignot, G. D. Cody, and A. R. Oganov, Inorg. Chem. 53(13), 7020-7027 (2014).

${ }^{5}$ D. Errandonea, Y. Meng, D. Hausermann, and T. Uchida, J. Phys. Condens. Matter 15(8), 1277 (2003).

${ }^{6}$ G. W. Stinton, S. G. MacLeod, H. Cynn, D. Errandonea, W. J. Evans, J. E. Proctor, Y. Meng, and M. I. McMahon, Phys. Rev. B 90(13), 134105 (2014).

${ }^{7}$ P. Hidnet and W. T. Sweeney, Bureau of Standards Journal of Research (United States Government Printing Office, Washington, 1929), Vol. 1, pp. 771-792.

${ }^{8}$ P. Lukac and A. Rudajevova, Kovove Mater. 41(5), 281-292 (2003), available at http://www.kovmat.sav.sk/issue.php?rr=41\&cc $=5$.

${ }^{9}$ P. D. Pathak and R. J. Desai, Phys. Status Solidi A 66, K179-K182 (1981).

${ }^{10}$ R. D. McCammon and G. K. White, Philos. Mag. J. Theor. Exp. Appl. Phys. 11(114), 1125-1134 (1965).

${ }^{11}$ O. O. Kurakevych, Y. Le Godec, and V. L. Solozhenko, J. Phys. Conf. Ser. 653(1), 012080 (2015).

${ }^{12}$ O. O. Kurakevych, Y. Le Godec, and V. L. Solozhenko, J. Phys. Conf. Ser. 950, 042023 (2017) 
${ }^{13}$ O. O. Kurakevych and V. L. Solozhenko, J. Superhard Mater. 36(4), 270-278 (2014).

${ }^{14}$ O. L. Anderson and D. G. Isaak, J. Phys. Chem. Solids 54(2), 221-227 (1993).

${ }^{15}$ O. L. Anderson, H. Oda, A. Chopelas, and D. G. Isaak, Phys. Chem. Miner. 19(6), 369-380 (1993).

${ }^{16}$ P. I. Dorogokupets and A. Dewaele, High Press. Res. 27(4), 431-446 (2007).

${ }^{17}$ A. Kubo, Y. Wang, C. E. Runge, T. Uchida, B. Kiefer, N. Nishiyama, and T. S. Duffy, J. Phys. Chem. Solids 69(9), 2255-2260 (2008).

${ }^{18}$ Z. Jouini, O. O. Kurakevych, H. Moutaabbid, Y. Le Godec, M. Mezouar, and N. Guignot, J. Superhard Mater. 38(1), 66-70 (2016).

${ }^{19}$ O. O. Kurakevych and V. L. Solozhenko, Molecules 21(10), 1399 (2016).

${ }^{20}$ O. Shimomura, Physica B + C 139-140, 292-300 (1986).

${ }^{21}$ A. N. Baranov, V. L. Solozhenko, C. Lathe, V. Z. Turkevich, and Y. W. Park, Supercond. Sci. Technol. 16(10), 1147 (2003).

${ }^{22}$ J. Li, C. Hadidiacos, H.-K. Mao, Y. Fei, and R. J. Hemley, High Press. Res. 23(4), 389-401 (2003).

${ }^{23} \mathrm{~J}$. Guignard and W. A. Crichton, Rev. Sci. Instrum. 86(8), 085112 (2015).
${ }^{24}$ A. Courac, Y. Le Godec, C. Renero-Lecuna, H. Moutaabbid, R. Kumar, C. Coelho-Diogo, C. Gervais, and D. Portehault, Inorg. Chem. 58(16), 10822-10828 (2019).

${ }^{25}$ V. L. Solozhenko, O. O. Kurakevych, Y. Le Godec, and V. V. Brazhkin, J. Phys. Chem. C 119(35), 20600-20605 (2015).

${ }^{26}$ R. R. Rao, Phys. Rev. B 10(10), 4173-4177 (1974).

${ }^{27}$ E. R. Naimon, Phys. Rev. B 4(12), 4291-4296 (1971).

${ }^{28}$ R. Srinivasan and R. Ramji Rao, J. Phys. Chem. Solids 32(8), 1769-1788 (1971).

${ }^{29}$ S. Anzellini, D. Errandonea, S. G. MacLeod, P. Botella, D. Daisenberger, J. M. De'Ath, J. Gonzalez-Platas, J. Ibáñez, M. I. McMahon, K. A. Munro, C. Popescu, J. Ruiz-Fuertes, and C. W. Wilson, Phys. Rev. Mater. 2(8), 083608 (2018).

${ }^{30}$ D. Errandonea, J. Appl. Phys. 108(3), 033517 (2010).

${ }^{31}$ L. V. Gurvich, I. V. Weits, V. A. Medvedev, V. S. Yungman, and G. A. Bergman, Thermodynamic Properties of Individual Substances (Nauka, Moscow, 1981).

${ }^{32}$ P. J. McGonigal, A. D. Kirshenbaum, and A. V. Grosse, J. Phys. Chem. 66(4), 737-740 (1962).

${ }^{33}$ See http://moltensalt.org/references/static/downloads/pdf/element-salt-densities. pdf for the data on density of molten elements and representative salts. 